\title{
Day-Case Laparoscopic Cholecystectomy in Childhood: Outcomes from a Clinical Care Pathway
}

\author{
Girish Jawaheer $^{1}$ Kathryn Evans ${ }^{1}$ Ritchie Marcus ${ }^{2}$ \\ ${ }^{1}$ Department of Pediatric Surgery, Birmingham Children's Hospital, \\ Birmingham, United Kingdom \\ 2 Department of Pediatric Anesthesia, Birmingham Children's \\ Hospital, Birmingham, United Kingdom \\ Address for correspondence and reprint requests Girish Jawaheer, \\ Department of Pediatric Surgery, Birmingham Children's Hospital, \\ Steelhouse Lane, Birmingham B4 6NH, United Kingdom \\ (e-mail: girish.jawaheer@bch.nhs.uk).
}

Eur J Pediatr Surg 2013;23:57-62.

\begin{abstract}
Keywords

- cholecystectomy

- laparoscopic

- clinical care pathway

- children

- day-case

Aim The aim of this study was to report clinical outcomes following the use of a pediatric day-case laparoscopic cholecystectomy (DCLC) clinical care pathway. The pathway was modified in September 2009 and we compare the clinical outcomes before and after this modification.

Methods A care pathway for DCLC was introduced in 2008 with emphasis on the day of admission, timing of surgery, choice of anesthetic agents, analgesia, postoperative feeding, mobilization, and pain scoring. Demographic and clinical data were recorded prospectively from January 2008 to January 2012. In September 2009, two modifications were made to the pathway. Induction of anesthesia was changed to total intravenous anesthesia, using propofol (target 4 to $6 \mu \mathrm{g} / \mathrm{mL}$ ) and remifentanil (target 3 to $5 \mathrm{ng} / \mathrm{mL}$ ) and the use of the gaseous anesthetic sevoflurane was eliminated with the aim of reducing the risk of postoperative nausea and vomiting (PONV). The postoperative feeding regime was changed from unrestricted to light diet for 72 hours. The rest of the pathway was unchanged. Data before (group 1) and following the modifications (group 2) were compared.

Results We admitted 25 children with symptomatic cholelithiasis for DCLC under the care of one surgeon: 12 in group 1 and 13 in group 2 . There were no significant differences in age between group 1 (median 13 [range 6 to 15] years) and group 2 (median 15 [9 to 16$]$ years) $(p=0.07)$. There were no intra- or postoperative complications. The day-case rate increased from $6 / 12$ (50\%) in group 1 to $12 / 13$ $(92 \%)$ in group $2(p=0.03)$. The incidence of PONV reduced from $7 / 12(58 \%)$ in group 1 to $0 / 13$ in group $2(p=0.002)$. PONV in group 1 resulted in overnight stay $(n=6)$ and readmission $(n=1)$. One patient in group 2 had an overnight stay due to poor mobilization.

Conclusions Adoption of a DCLC pathway is feasible and safe for children. Emphasis on adequate pain management and avoidance of PONV results in a high rate of day-case surgery equivalent to that achieved in adult practice.
\end{abstract}

received

May 17, 2012

accepted after revision

August 18, 2012

published online

November 19, 2012 (c) 2013 Georg Thieme Verlag KG

Stuttgart · New York
DOI http://dx.doi.org/

10.1055/s-0032-1329701.

ISSN 0939-7248. 


\section{Introduction}

The role of laparoscopic cholecystectomy (LC) as first line treatment for symptomatic cholelithiasis is now undisputed. A relatively recent development has been the introduction of day-case LC (DCLC) in adult practice in the 1990s and there is an abundance of reports promoting its use. ${ }^{1-15}$ DCLC is now listed on the British Association of Day Surgery ${ }^{16}$ and the Audit Commission ${ }^{17}$ list of procedures. Important considerations in determining therapeutic modality in the 21st century are clinical outcomes, patient choice and satisfaction, and cost-effectiveness. Patient satisfaction rates of 92 to $95 \%$ have been reported. ${ }^{1,4,5,18,19}$ The primary consideration however should remain patient safety. A recent meta-analysis of five randomized controlled trials in adults demonstrated that compared with overnight stay, DCLC is safe and effective in selected patients and is likely to save costs. ${ }^{20}$ The role of DCLC in pediatric practice is yet to be established. There has been only one report on DCLC in children. ${ }^{21}$ One possible reason for this discrepancy is the lack of clear practice guidelines or care pathways in children. In contrast, there is a published guideline on DCLC from the British Association of Day Surgery in adults. ${ }^{22}$ There is growing evidence to suggest that clinical care pathways improve outcomes and reduce hospital stay in adults $^{23,24}$ and children. ${ }^{25,26}$ There are presently no published guidelines or pathways on DCLC in children. The aim of this article is to address this void in the literature and to report clinical outcomes following the use of a pediatric DCLC clinical care pathway at our institution. The pathway was modified in September 2009 and we compare the clinical outcomes before and after this modification.

\section{Patients and Methods}

All LCs in the Pediatric Surgical Department are performed by a single surgeon. In December 2007, a clinical care pathway was introduced following an audit of 36 LCs performed between September 2003 and September 2007. The essential elements of the pathway are outlined in -Fig. 1. The changes in practice following the audit were admission on the procedure day, the cessation of blood investigations including group and save and blood cross-matching, a standardized anesthetic protocol, discontinuation of the use of routine antibiotics, minimization of the use of intravenous morphine in the postoperative period and pain scoring using the Wong and Baker Visual FACES pain rating scale (Wong-Baker Foundation, Oklahoma City, OK, USA). ${ }^{27}$ Exclusion criteria for DCLC in patients undergoing LC are listed in -Table 1.

All patients were seen in the outpatient clinic before DCLC and informed consent was obtained. Blood tests and an abdominal ultrasound scan were requested. Patients were all admitted electively on the morning of the procedure and underwent a LC on the morning list. All patients were anesthetized by consultant pediatric anesthetists. Before September 2009, induction of anesthesia was either gaseous with sevoflurane or intravenous with propofol. All patients were ventilated via an endotracheal tube with air, oxygen, and isoflurane. Intraoperative analgesia comprised either intravenous fentanyl up to $5 \mu \mathrm{g} / \mathrm{kg}$ in incremental doses or (in two patients) a remifentanil infusion with an intravenous morphine bolus of 0.1 to $0.15 \mathrm{mg} / \mathrm{kg}$ toward the end of surgery. In addition, all patients received intraoperative diclofenac and intravenous paracetamol. All patients had infiltration of $0.25 \%$ levobupivacaine hydrochloride at port sites before port insertion. Prophylactic antiemesis was provided by both dexamethasone and ondansetron. All patients received $10 \mathrm{~mL} / \mathrm{kg}$ of Hartmann's solution over 30 minutes after induction of anesthesia followed by an infusion of $5 \mathrm{~mL} / \mathrm{kg} / \mathrm{h}$ intraoperatively. Postoperatively, all patients received regular paracetamol, codeine, and nonsteroidal antiinflammatory drug. "Oramorph" was prescribed for rescue analgesia and ondansetron for antiemesis. After September 2009, the anesthetic technique was changed to total intravenous anesthesia, using propofol (target 4 to $6 \mu \mathrm{g} / \mathrm{mL}$ ) and remifentanil (target 3 to $5 \mathrm{ng} / \mathrm{mL}$ ) delivered by Alaris PK pumps (CareFusion, San Diego, CA, USA) and the use of the gaseous anesthetic sevoflurane was eliminated with the aim of reducing the risk of postoperative nausea and vomiting (PONV). An intravenous morphine bolus of 0.1 to $0.15 \mathrm{mg} / \mathrm{kg}$ was given toward the end of the surgery. The other modification was a change in the postoperative feeding regime from unrestricted to light diet for 72 hours. The latter consisted of frequent small meals such as bread, cereals, soup, fruit, and yogurt and avoidance of large meals and fried foods. Other analgesia, antiemesis, and fluid management were unchanged.

A standard four-port technique was used with a 30-degree $10 \mathrm{~mm}$ port at the umbilicus and three $5 \mathrm{~mm}$ secondary ports. Carbon dioxide $\left(\mathrm{CO}_{2}\right)$ insufflation pressures were kept at or below $12 \mathrm{~mm} \mathrm{Hg}$ and a low insufflation rate of $1 \mathrm{~L} / \mathrm{min}$ was used. An intraoperative cholangiogram was performed routinely. At the end of the procedure, an effort was made to evacuate as much $\mathrm{CO}_{2}$ from the peritoneal cavity as possible. In the postoperative period, early mobilization was encouraged as well as enteral intake of fluid and light diet. Pain was assessed by the child's nurse and scored using the Wong and Baker FACES pain rating scale. All patients were seen at the end of the afternoon by the anesthetic team and jointly by the surgical and the nursing team and a decision was made regarding discharge. It was considered important to leave the final decision regarding discharge from hospital to be made jointly by the patients' families and the nursing team. Criteria for discharge were normal temperature, pulse and blood pressure, tolerance of fluid and light diet, adequate pain control, comfortable mobilization, and patient/carer satisfaction with discharge. The carers were provided with the ward contact details and encouraged to have a low threshold for calling for advice. The following day, the carers were telephoned by the surgeon and a pain score was obtained.

All LCs performed between January 2008 and October 2011 were entered on an electronic database, and demographic and clinical data including operative details and outcomes and intra- and postoperative complications were recorded prospectively. Patients were placed in two groups: group 1 included patients from pathway introduction in January 2008 to September 2009 when the pathway was modified and group 2 comprised patients postpathway modification from 
Referral of children with symptomatic cholelithiasis to outpatients clinic

History and examination

Informed consent for laparoscopic cholecystectomy and intraoperative cholangiogram

\section{Outpatient FBC, U\&Es, LFTs and abdominal ultrasound scan}

Inclusion criteria for DCLC met-DCLC
scheduled $\begin{gathered}\text { Inclusion criteria for DCLC not met- } \\ \text { DCLC not appropriate }\end{gathered}$

Admission on morning of procedure and LC on morning list

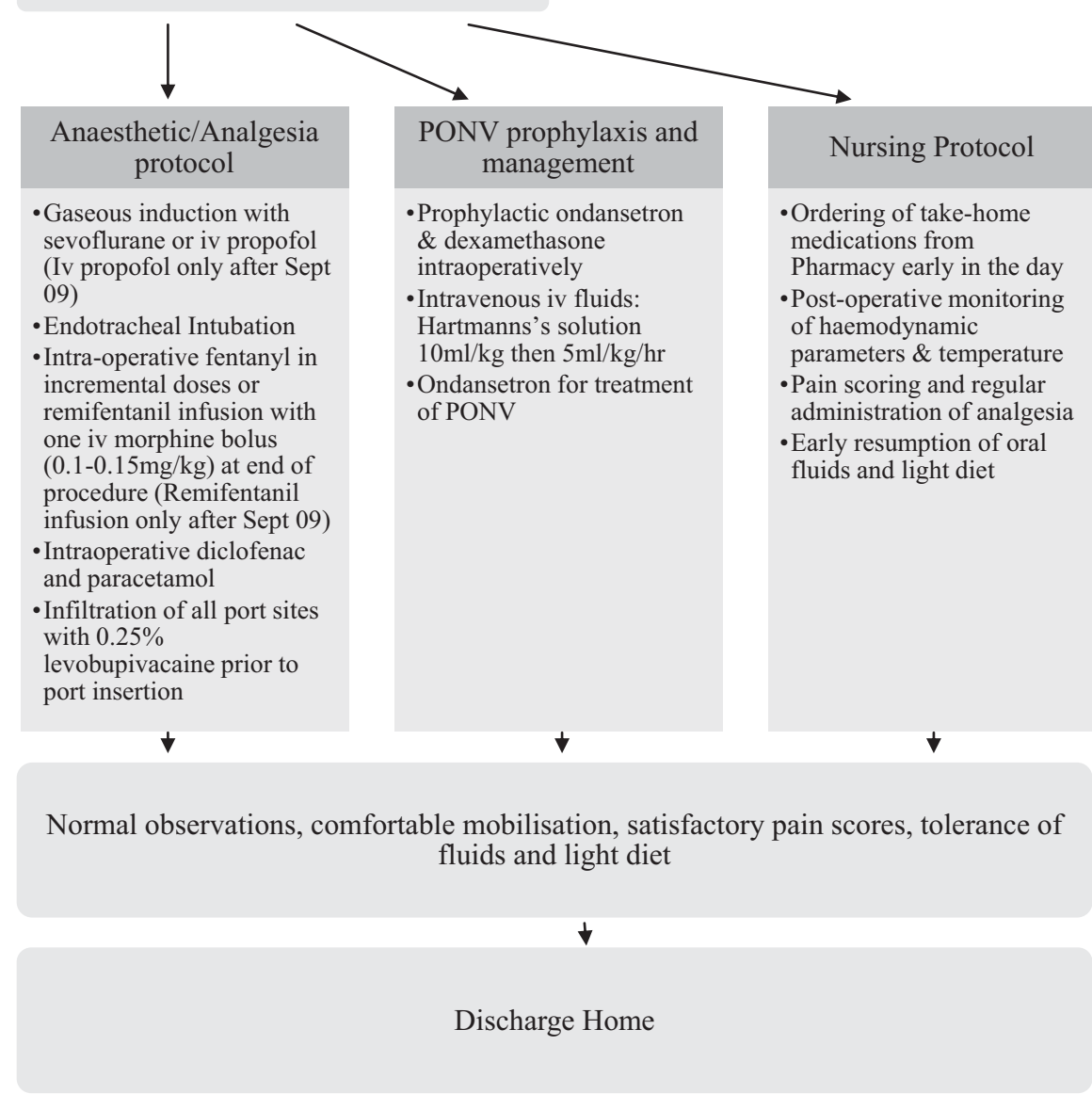

Fig. 1 Clinical care pathway for children undergoing DCLC. DCLC, day-case laparoscopic cholecystectomy; FBC, full blood count; LFTs, liver function tests; U\&Es, urea and electrolytes.

September 2009 to October 2011. All patients were followed up in the outpatient clinic 6 weeks following discharge.

Statistical analyses were performed using the $t$ test to compare age and weight and the Fisher exact test to compare the rate of PONV and the rate of day-case surgery. Results were considered to be significant at $p \leq 0.05$.

The introduction of the clinical care pathway was in line with our institutional policies. The fundamental elements of the pathway were based on the outcome of an audit regis- tered with our Governance Department. Ethics approval was not required as the pathway contained treatment modalities which are well established within clinical practice.

\section{Results}

Clinical outcomes are reported in 25 children: 12 in group 1 and 13 in group 2. Between January 2008 and April 2009, out of 13 children having LC as the sole procedure, 12 (92\%) 
Table 1 Exclusion criteria for DCLC

\begin{tabular}{|l|}
\hline Sickle cell disease \\
\hline Abnormal liver function \\
\hline Presence of comorbidity \\
\hline Performance of other operations under the same anesthetic \\
\hline Presence of adverse social conditions at home \\
\hline Long distance from hospital \\
\hline
\end{tabular}

Abbreviation: DCLC, day-case laparoscopic cholecystectomy.

children ( 7 girls and 5 boys) met the criteria to undergo DCLC (group 1). Median age was 13 (range 6 to 15) years and median weight was 51 (range 18 to 104) $\mathrm{kg}$. Indications for surgery were biliary colic $(n=9)$ and gallstone pancreatitis $(n=3)$. One child was excluded due to the presence of comorbidity (sickle cell anemia). Between September 2009 and October 2011, out of 16 children having solely LC, 13 ( $81 \%)$ children (10 girls and 3 boys) met the criteria for DCLC (group 2). Median age was 15 (9 to 16) years and median weight was 55.9 (range 27.2 to 77.5 ) $\mathrm{kg}$. There were no significant differences in age or weight between the two groups ( $p=0.07$ and $p=0.52$, respectively). Indications for surgery were biliary colic $(n=10)$ and gallstone pancreatitis $(n=3)$. Three children were excluded due to the presence of comorbidity (nephrotic syndrome, $n=1$ ) and living a long distance away from the hospital $(n=2)$. Liver function tests were normal in all patients at the time of surgery. The diagnosis of cholelithiasis was confirmed by ultrasonography in all patients before admission. Median operating time was 90 (range 70 to 150) minutes. Intraoperative cholangiography excluded the presence of common bile duct stones or obstruction in all the patients. Seven (58\%) children in group 1 had a dilated biliary system, with a median common bile duct diameter of 5.6 (range 4 to 18 ) $\mathrm{mm}$. Three children in group 2 had a dilated biliary system with a median CBD diameter $9 \mathrm{~mm}$ (range 6 to $10 \mathrm{~mm}$ ). There were no intra- or postoperative minor or major complications and there were no conversions to open procedure. There was no intraoperative bleeding which required a blood transfusion. Six (50\%) children in group 1 were discharged on the day of surgery. In this group, median pain score was $3 / 10$ (range 0 to 3 ) before discharge and 3 (range 2 to 4 ) on the day after the procedure. Five children had an overnight stay and one child had a twonight stay. Reasons for the delay in discharge were PONV in all the six cases. There was no delay in discharge secondary to pain. One of the six patients discharged on the day of surgery was readmitted 2 days later with PONV. In group 2, 12 (92\%) of the patients were discharged on the day of surgery. In this group, median pain score was $2 / 10$ (range 0 to 4 ) on the day of discharge and $1 / 10$ (range 0 to 4 ) on the day after the procedure. The postdischarge pain scores were significantly less in group $2(p=0.04)$. The incidence of PONV reduced from $7 / 12(58 \%)$ in group 1 to $0 / 13$ in group $2(p=0.002)$ following pathway modification. One patient in group 2 had an overnight stay due to poor mobilization. Comparisons between groups 1 and 2 are summarized in -Table 2. All patients were seen in the outpatient clinic 6 weeks following discharge. There was universal patient and carer satisfaction with the patient journey through the pathway.

\section{Discussion}

This is the first report of DCLC in childhood to be based on a clear, detailed, and reproducible clinical care pathway. It is of interest that $50 \%$ of the patients in group 1 admitted for DCLC were discharged on the procedure day and this increased significantly to $92 \%$ in group $2(p=0.003)$ following our pathway modification. This compares favorably with adult practice where early experience with DCLC achieved sameday discharge rate of $56 \% 28$ and the rate has since risen to 65 to $94 \%{ }^{4-6,19,29,30}$

The success of a DCLC service is dependent on four crucial factors: a multidisciplinary team approach, rigorous patient selection, adequate pain management, and avoidance of PONV. Each one of these factors will now be discussed in more detail.

LC was introduced in our department in 2003 and a policy was instituted for all LCs to be performed by a single surgeon. The latter initially worked in close conjunction with a single consultant pediatric anesthetist. This set-up led to $\sim 10$ LCs being performed by a single team and invaluable experience was accumulated in a relatively short space of time. This accounted for the absence of minor or major complications and conversions to open in this series which compares favorably with adult series where a minor complication

Table 2 Comparison of demographic and clinical data in groups 1 and 2

\begin{tabular}{|l|l|l|l|}
\hline & Group 1 & Group 2 & $p$ value \\
\hline Age, median (range) years & $13(6-15)$ & $15(9-16)$ & 0.07 \\
\hline Weight, median (range) kg & $51(18-104)$ & $55.9(27.2-77.5)$ & 0.52 \\
\hline Pain score predischarge & $3(0-3)$ & $2(0-4)$ & 0.34 \\
\hline Pain score postdischarge & $3(2-4)$ & $1(0-4)$ & 0.04 \\
\hline PONV & $7(58 \%)$ & 0 & 0.002 \\
\hline Day-case rate & $6 / 12(50 \%)$ & $12 / 13(92 \%)$ & 0.03 \\
\hline
\end{tabular}

Abbreviation: PONV, postoperative nausea and vomiting. 
rate of $2 \%$ and a major complication rate of $5 \%{ }^{1}$ and a conversion rate of $2 \%^{6}$ have been reported. Data relating to the cases were collected prospectively and clinical outcomes were audited. The most significant lessons to be learnt by the audit were that admission on the day before the procedure was unnecessary, that explanation of the procedure and discharge policy to the families in the outpatient setting was extremely important in shaping their expectations and influencing their acceptance of having a major procedure performed in an ambulatory setting, and that the use of intravenous opioids, as patient-controlled analgesia (PCA), in the postoperative setting introduced significant delays to discharge. The role of the nursing team in achieving one's goals should not be underestimated. The postoperative nursing management of children having DCLC requires a major shift away from a traditional conservative approach with regard to introduction of enteral feeds, mobilization, and pain management. In our experience, an important role of the nurse is to introduce an element of balance to the decision regarding patient discharge. It is conceivable that if the decision regarding discharge is made solely by the surgeons, some patients may be discharged prematurely to bolster their same-day discharge rates in spite of high levels of pain or difficulties with mobilization or nausea. To eliminate this bias, the final decision for discharge was made by the nurse caring for the patient in close conjunction with the family. There were several instances in group 1 where the patients had an overnight stay purely because the nurses were not comfortable with same-day discharge. It is not known whether these patients could have been managed successfully at home.

Rigorous patient selection is a prerequisite for the success of DCLC. All the patients in our series were teenagers except for three who were aged 6 and two who were 9 years old. Of these three children, only one of the 9 years old achieved same-day discharge. This series is too small for one to be able to make firm conclusions but younger age may be found to be a relative contraindication in the future. One possibility is that these younger children are less self-motivated for same-day discharge. Patients with sickle cell disease were excluded because of the need for adequate hydration and their dependence upon intravenous fluids until a full oral intake is achieved. An interesting group of patients were those with a dilated common bile duct. This constituted an exclusion criterion in the British Association of Day Surgery publication. ${ }^{22}$ Of the 18 patients, 5 who had same-day discharge had a dilated common bile duct. Ten patients in the whole series had biliary dilatation but none of them had bile duct stones or obstruction shown on cholangiography. We therefore conclude that as long as liver function tests are normal, a dilated biliary system does not constitute an exclusion criterion for DCLC in children. Interestingly, a recent publication found that the need for laparoscopic bile duct exploration at the time of LC in adults did not influence same-day discharge. ${ }^{15}$

A cornerstone of DCLC practice is adequate pain management. An interesting but surprising finding of our audit was that discharge following LC was delayed in those patients who had morphine delivered via a patient-controlled device. This finding concurs with adult practice where a randomized controlled trial investigating the role of opioid PCA found that PCA was associated with a significantly higher incidence of PONV. ${ }^{31}$ The intraoperative use of intravenous paracetamol and diclofenac reduced the need for opioid analgesia. A randomized controlled trial demonstrated that infiltration of port sites with levobupivacaine before port insertion is associated with less postoperative analgesic consumption. ${ }^{32}$ We did not use intraperitoneal local anesthetic in this series but this is worth considering in the future given the recent randomized sham controlled trial demonstrating benefit from intraperitoneal techniques. ${ }^{33}$ During postoperative recovery, we would emphasize that the combination of paracetamol, codeine, and nonsteroidal anti-inflammatory agent be prescribed in the "regular" section of the prescription chart instead of the "PRN" section because we found the latter was associated with reactive and therefore insufficient administration of the drugs by nursing staff. It has been reported that a 2-day supply of take-home analgesia is insufficient in adult practice. ${ }^{13}$ We would recommend that the take-home pack of analgesics should contain a minimum supply of 5 days of analgesia. The reason for the significantly lower pain scores in group 2 in unclear.

The biggest challenge to performing LC in children is PONV. PONV accounted for all delays in discharge in our series with an incidence as high as $58 \%$ in group 1 . A similar experience has been noted in adult series with a high incidence of PONV of $25 \%{ }^{13}$ It is important for appropriate measures to be put in place to minimize its incidence. Our current protocol includes such measures as adequate intraoperative hydration, routine combination intraoperative antiemetics, minimization of the use of long acting intravenous opioids, and a strict dietary regime which includes only light meals for the first few days following the procedure. The use of a propofol-based anesthetic technique has been shown to reduce the incidence of PONV in adults undergoing $\mathrm{LC}^{34}$ and children having strabismus surgery. ${ }^{35}$ In children undergoing correction of prominent ears, the use of propofolbased anesthesia has both decreased the incidence of PONV and increased the number of children fit for discharge on the day of surgery. ${ }^{36}$ However, the use of thiopental for induction in the volatile anesthetic group in this study would have caused additional sedation and PONV and may have affected the results. Our series shows a significant reduction in PONV following the use of a total intravenous anesthesia technique using propofol and remifentanil from 58 to $0 \%$. We do not prescribe antiemetics regularly postoperatively nor do we include them in the take-home pack due to the low rate of PONV and some evidence from adult practice that this is of little benefit. $^{37}$

The data provided in this report demonstrate that DCLC in children is feasible in the majority of patients requiring cholecystectomy as a sole procedure and can be performed with excellent results without compromising patient safety. A multidisciplinary team approach and the adoption of a clinical care pathway focusing on adequate pain management and avoidance of PONV are a prerequisite for success. 
Conflict of Interest

None

\section{References}

1 Mjåland O, Raeder J, Aasboe V, Trondsen E, Buanes T. Outpatient laparoscopic cholecystectomy. Br J Surg 1997;84(7):958-961

2 Campanelli G, Cavagnoli R, Cioffi U, De Simone M, Fabbiani M, Pietri P. Can laparoscopic cholecystectomy be a day surgery procedure? Hepatogastroenterology 1998;45(23):1422-1429

3 Kiely JM, Brannigan AE, Foley E, Cheema S, O’Brien W, Delaney PV. Day case laparoscopic cholecystectomy is feasible. Ir J Med Sci 2001;170(2):98-99

4 Ammori BJ, Davides D, Vezakis A, et al. Day-case laparoscopic cholecystectomy: a prospective evaluation of a 6 -year experience. J Hepatobiliary Pancreat Surg 2003;10(4):303-308

5 Leeder PC, Matthews T, Krzeminska K, Dehn TC. Routine day-case laparoscopic cholecystectomy. Br J Surg 2004;91(3):312-316

6 Johansson M, Thune A, Nelvin L, Lundell L. Randomized clinical trial of day-care versus overnight-stay laparoscopic cholecystectomy. Br J Surg 2006;93(1):40-45

7 Metcalfe MS, Mullin EJ, Maddern GJ. Relaxation of the criteria for day surgery laparoscopic cholecystectomy. ANZ J Surg 2006;76 (3):142-144

8 Kasem A, Paix A, Grandy-Smith S, El-Hasani S. Is laparoscopic cholecystectomy safe and acceptable as a day case procedure? J Laparoendosc Adv Surg Tech A 2006;16(4):365-368

9 Berrevoet E, Biglari M, Sinove Y, De Baardemaeker L, Troisi R, de Hemptinne B. Outpatient laparoscopic cholecystectomy in Belgium: what are we waiting for? Acta Chir Belg 2006;106(5): 537-540

10 Victorzon M, Tolonen P, Vuorialho T. Day-case laparoscopic cholecystectomy: treatment of choice for selected patients? Surg Endosc 2007;21(1):70-73

11 Proske JM, Dagher I, Revitea C, et al. Day-case laparoscopic cholecystectomy: results of 211 consecutive patients. Gastroenterol Clin Biol 2007;31(4):421-424

12 Rathore MA, Andrabi SI, Mansha M, Brown MG. Day case laparoscopic cholecystectomy is safe and feasible: a case controlled study. Int J Surg 2007;5(4):255-259

13 Kavanagh T, Hu P, Minogue S. Daycase laparoscopic cholecystectomy: a prospective study of post-discharge pain, analgesic and antiemetic requirements. Ir J Med Sci 2008;177(2):111-115

14 Cassinotti E, Colombo EM, Di Giuseppe M, Rovera F, Dionigi G, Boni L. Current indications for laparoscopy in day-case surgery. Int J Surg 2008;6(6):(Suppl 1):S93-S96

15 Kow AW, Tan A, Chan SP, et al. An audit of ambulatory laparoscopic cholecystectomy in a Singapore institution: are we ready for daycase laparoscopic cholecystectomy? HPB (Oxford) 2008;10(6): 433-438

16 Cahill CJ. Basket cases and trolleys. Day surgery proposals for the Millenium. Journal of One-Day Surgery 1999;9(1):11-12

17 Audit Commission. Day Surgery: Review of National Findings. London: Audit Commission Publications; 2001

18 Psaila J, Agrawal S, Fountain U, et al. Day-surgery laparoscopic cholecystectomy: factors influencing same-day discharge. World J Surg 2008;32(1):76-81

19 Briggs CD, Irving GB, Mann CD, et al. Introduction of a day-case laparoscopic cholecystectomy service in the UK: a critical analysis of factors influencing same-day discharge and contact with primary care providers. Ann R Coll Surg Engl 2009;91(7):583-590

20 Gurusamy KS, Junnarkar S, Farouk M, et al. Day-case versus overnight stay for laparoscopic cholecystectomy. Cochrane Database Syst Rev 2008;3(CD006798):1469-1493

21 Méndez K, Sabater R, Chinea E, Lugo-Vicente H. Is there a safe advantage in performing outpatient laparoscopic cholecystectomy in children? J Pediatr Surg 2007;42(8):1333-1336

22 McWhinnie D, Ellams J, Cahill J, et al. Daycase laparoscopic cholecystectomy. Available at: http://www.daysurgeryuk.net/ bads/joomla/files/Handbooks/LaparoscopicCholecystectomy.pdf

23 Chase DM, Lopez S, Nguyen C, Pugmire GA, Monk BJ. A clinical pathway for postoperative management and early patient discharge: does it work in gynecologic oncology? Am J Obstet Gynecol 2008;199(5):541, e1-e7

24 Joh YG, Lindsetmo RO, Stulberg J, Obias V, Champagne B, Delaney CP. Standardized postoperative pathway: accelerating recovery after ileostomy closure. Dis Colon Rectum 2008;51(12): 1786-1789

25 Almond SL, Roberts M, Joesbury V, et al. It is not what you do, it is the way that you do it: impact of a care pathway for appendicitis. J Pediatr Surg 2008;43(2):315-319

26 Emil S, Taylor M, Ndiforchu F, Nguyen N. What are the true advantages of a pediatric appendicitis clinical pathway? Am Surg 2006;72(10):885-889

27 Wong DL, Baker CM. Pain in children: comparison of assessment scales. Pediatr Nurs 1988;14(1):9-17

28 Huang A, Stinchcombe C, Davis M, et al. Prospective five-year audit for day-case laparoscopic cholecystectomy. Journal of One-Day Surgery 2000;9(4):15-17

29 Akoh JA, Watson WA, Bourne TP. Day case laparoscopic cholecystectomy: reducing the admission rate. Int J Surg 2011;9(1):63-67

30 Patel SD, Patel H, Ganapathi S, Marshall N. Day case laparoscopic cholecystectomy carried out using the harmonic scalpel: analysis of a standard procedure. Surg Laparosc Endosc Percutan Tech 2010;20(1):20-23

31 Sinha S, Munikrishnan V, Montgomery J, Mitchell SJ. The impact of patient-controlled analgesia on laparoscopic cholecystectomy. Ann R Coll Surg Engl 2007;89(4):374-378

32 Cantore F, Boni L, Di Giuseppe M, Giavarini L, Rovera F, Dionigi G. Pre-incision local infiltration with levobupivacaine reduces pain and analgesic consumption after laparoscopic cholecystectomy: a new device for day-case procedure. Int J Surg 2008;6(Suppl 1): S89-S92

33 Roberts KJ, Gilmour J, Pande R, Nightingale P, Tan LC, Khan S. Efficacy of intraperitoneal local anaesthetic techniques during laparoscopic cholecystectomy. Surg Endosc 2011;25(11):3698-3705

34 Raeder JC, Mjåland O, Aasbø V, Grøgaard B, Buanes T. Desflurane versus propofol maintenance for outpatient laparoscopic cholecystectomy. Acta Anaesthesiol Scand 1998;42(1):106-110

35 Gürkan Y, Kiliçkan L, Toker K. Propofol-nitrous oxide versus sevoflurane-nitrous oxide for strabismus surgery in children. Paediatr Anaesth 1999;9(6):495-499

36 Woodward WM, Barker I, John RE, Peacock JE. Propofol infusion vs thiopentone/isoflurane anaesthesia for prominent ear correction in children. Paediatr Anaesth 1997;7(5):379-383

37 Thagaard KS, Steine S, Raeder J. Ondansetron disintegrating tablets of $8 \mathrm{mg}$ twice a day for 3 days did not reduce the incidence of nausea or vomiting after laparoscopic surgery. Eur J Anaesthesiol 2003;20(2):153-157 